NASA CONTRACTOR REPORT

0
0
0
0
0
$\pi$
$\vdots$
2

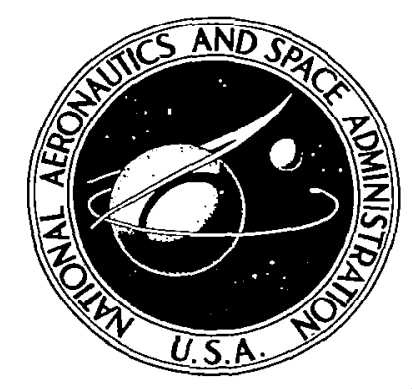

NASA CR-91部

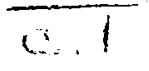

[OAN CONY: TH:

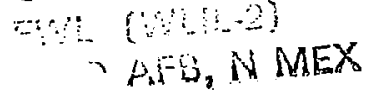

\title{
INFRARED OBSERVATIONS OF THE OUTER SOLAR CORONA
}

by Robert M. MacQueen

Prepared by

THE JOHNS HOPKINS UNIVERSITY

Baltimore, Md.

for

NATIONAL AERONAUTICS AND SPACE ADMINISTRATION • WASHINGTON, D. C. - APRIL 1968 


\title{
INFRARED OBSERVATIONS OF THE OUTER SOLAR CORONA
}

\author{
By Robert M. MacQueen
}

Distribution of this report is provided in the interest of information exchange. Responsibility for the contents resides in the author or organization that prepared it.

Prepared under Grant No. NGR-21-001-044 by

THE JOHNS HOPKINS UNIVERSITY

Baltimore, Md.

for

\section{NATIONAL AERONAUTICS AND SPACE ADMINISTRATION}

For sale by the Clearinghouse for Federal Scientific and Technical Information Springfield, Virginia 22151 - CFSTI price $\$ 3.00$ 
FOREWORD

The National Aeronaut1cs and Space Administration Grant NGR-21-001-044 supported the costs of design and construction of an Infrared telescope to measure infrared emission from circumsolar dust during the November 12, 1966 eclipse.

The grant supported the Johns Hopkins University Eclipse Expedition to Bolivia, except for Mr. MacQueen's salary.

The grant supported the design and construction of a balloonborne coronagraph, and certain necessary modifications of our balloon-borne Bal-Ast equipment, as well as the balloon field trips to Palestine, Texas, that culminated in the successful flight of January 9, 1967.

The purchase of certain items of optical equipment was provided for by ARPA Grant DA-ARO-D-31-124-G756.

The development and testing of the experimental ideas for the balloon-borne coronagraph and infrared telescope were supported by OSR Contract AF $49(638)-1432$.

The subjoined material describes the developed equipment and results obtained with it.

John Strong 
ABSTRACT

The $2.2 \mu$ wavelength infrared outer solar corona has been observed in two experiments: first, employing a ground-based telescope from a high altitude site near Pulacayo, Bolivia, during the 93 second totality of the eclipse of 12 November 1966; and second, employing a stratospheric balloon package with an infrared coronagraph, flown to an altitude of $28 \mathrm{~km}$, during a 5.25 hour period, on 9 January 1967.

The design and evaluation of the infrared balloon-borne coronagraph is discussed in detail, and the various origins of stray light investigated. The completed flight coronagraph was found to Introduce a stray radiance of less than $3 \times 10^{-12} \mathrm{~B}$, where $B_{\odot}$ is the radiance of the mean solar disk at wavelength $2.2 \mu$. During eclipse the corona was observed from $2 R_{\odot}$ to $6 R_{\odot}$ in the ecliptic plane, and at a single point perpendicular to the ecliptic at $5 R_{\odot} \cdot$ Balloon observations covered the range $3.7 R_{\odot}$ to $10 R_{\odot}$, at position angles from $20^{\circ}$ north to $40^{\circ}$ south of the ecliptic, on the east $11 \mathrm{mb}$ of the sun.

Features attributed to the vaporization of interplanetary material were observed at $3.5 \mathrm{R}_{\odot}, 4 \mathrm{R}_{\odot}, 8.7 \mathrm{R}_{\odot}$, and $9.2 \mathrm{R}_{\odot}$, the former two during the period of totality, and the latter three during the balloon observations. 


\section{ACKNOWLEDGEMENTS}

We wish to express our indebtedness to the National Aeronautics and Space Administration whose financial support of this program made possible the work and results reported herein.

J. F. McClellan and F. H. Owens were responsible for the mechanical design of the balloon instrument, and $R$. W. McNiel the electronic design. We also acknowledge the aid of D. I. McLeod and W. S. Dalton under trying field conditions in Bolivia; the latter was also responsible for most of the mechanical construction of the eclipse telescope and balloon coronagraph. Electronic construction was under the competent supervision of W. T. Green. Balloon tracking responsibilities in the field were handled by W. G. Mankin; he is also responsible for several computer programs used during the course of this work, and helpful discussions.

Further, we thank Dr. H. S. Ahluwalhia and Fernando Sheriph of the Laboratorio de Fisica Cosmica, Universidad Mayor de San Andres, La Paz, Bolivia, for invaluable aid in all phases of the eclipse expedition. D. K. Watson of the High Altitude Observatory was instrumental in planning details of the expedition, and we also recognize the coordinating actions of Dr. Robert Fleischer, Deputy Head, Office of International Science Activities, National Science Foundation. 
The competence of the staff at the National Center for Atmospheric Research Balloon Launch Facility was outstanding, and in particular we are grateful to Operations Supervisor
A1 Shipley for patient counsel during the two months at the Palestine, Texas installation.
We thank Dr. Gordon Newkirk, Jr. of the High Altitude
Observatory for his hospitality in allowing us to use the clean facility there for scattering measurements in February 1966, and also for his useful comments concerning the various phases of the work. Finally, we thank Mr. R. T. Hansen, also of the High Altitude Observatory, for supplying $\mathrm{K}$-coronameter data on the inner corona for the date of balloon flight. 
TABLE OF CONTENTS

page

Foreword

iii

Abstract

$\mathrm{v}$

Acknowledgements

vii

I. Introduction

1

II. The Infrared Coronagraph

4

III. Instrumentation and Calibrations 17

IV. Observational Results

31

Bibliography

35

Table

37

Figures

38 


\section{INTRODUCTION}

The study of the solar corona in the infrared spectral region has largely been unexploited in the past, partially due to the technological lag of infrared techniques as compared with those in the vistble region of the spectrum. Taylor and MacQueen (1964) briefly summarized the past infrared experiments on the corona; since that time there have been infrared studies of line emission from the inner corona (Mangus and Stockhausen 1966), but no studies of the continuum corona have been published, excepting a parallel study to this (Peterson 1967). Motivation for such studies has been provided by calculations of Harwit (1964) and Peterson $(1963,1964,1965)$ in investigations into the thermal emission of dust particles in interplanetary space. Their models are somewhat high in their prediction of the expected infrared radiances, because of the assumption that the interplanetary grains are black-bodies; however, when less favorable assumptions leading to considerably lower grain temperatures are employed in the emission model of Peterson (1963), the predicted radiances are well within the range of current technology. Further, recent work by Low and Smith (1966) on what is believed to be a pre-planetary system serves as an additional stimulus for work on the dust envelopes of stars.

Attempts to identify the infrared dust emission by examining the nearsolar daytime sky have been made by Lazarev (1964) and Moroz and Boiko (1965); such experiments are fraught with difficulties in attempting to see a phenomenon whose magnitude would only be about $1 \%$ of the normal background sky radiance level. Lazarev claimed detection of the infrared emission; however, Moroz and Boiko found no such evidence, with equipment 
capable of measuring signals several orders of magnitude smaller than those reported by Lazarev. The latter work led to estimates of the upper limit of the number and mass density of the interplanetary particles at 1 astronomical unit (AU).

The possibility of detection of thermal radiation from the particulate matter near the sun leads to the possibility of observation of the dustfree zone -- that zone about the sun where it has been supposed that interplanetary matter becomes vaporized because of intense insolation. The possibility of the existence of such a zone was first put forth by Anderson (1924), and led to calculations by Russell (1929) and Over (1958) as to possible temperatures encountered by the particles, and also estimates as to the size of the region of the probable evaporation sphere about the sun. The latter work on the vaporization of $\mathrm{SiO}_{2}$ spherical crystals, led to an estimate of $4 \mathrm{R}_{\rho}$ as the boundary of the probable dust-free zone. Over pointed out the estimate of the vaporization zone position would necessarily be altered if the crystals were blacker than he assumed.

That the vaporization has not been seen in past coronal observations, and would be more likely to be seen through infrared emission observations, is due principally to two reasons; first, the emission process is probably isotropic, or nearly so, while the scattering is highly anisotropic in the forward direction. So visible wavelength observations of the corona at, say, an elongation angle corresponding to $4 \mathrm{R}_{\mathrm{e}}$ are heavily weighted with the contribution of scattering of material actually nearer the observer, and hence at greater sun-particle distances. Second, the effective cross-section of the scattering process is independent of the position of the particle relative to the sun, while for emission, the process is dependent upon the 
temperature of the grain, which, in turn, is a function of the solargrain distance. The first point is illustrated by the calculations of Ingham (1961), who found that the effect of a dust-free zone at $4 R_{e}$ on his model corona was to alter the visible coronal intensity beyond about

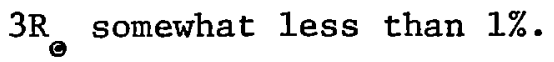

Current work on the dynamics of particles in interplanetary space has led to important questions concerning the fate of the particulate matter; Belton (1967) has suggested that the particles partially evaporate, with this onset of vaporization effectively stabilizing the dust orbit, after which the material moves away from the sun. Finally, Kaiser (1967a, 1967b) has examined a model of thermal emission of quartz particles in which the particles are subdivided by their optical sizes, and has found that such a procedure can be made to reasonably fit the observations herein presented.

Observations of the outer solar corona at a wavelength of $2.2 \mu$ have been carried out during the total solar eclipse of 12 November 1966 from a ground-based site near Pulacayo, Bolivia, with an infrared telescope, and also during a stratospheric balloon flight of 9 January 1967, employing an infrared coronagraph.

We shall examine in some detail the design and evaluation of the infrared coronagraph in Section II, while Section III will describe the eclipse and balloon instrumentation and calibrations. Section IV will present the results of the eclipse and balloon observations. 


\section{I.I. THE INFRARED CORONAGRAPH}

\section{Introduction}

From the time of B. Lyot's pioneer work (Lyot 1930, 1931, 1939) the coronagraph art has progressed to the stage where the inner corona (to about $1.1 R_{\odot}$, measured from the center of the solar disk) is observed on a regular basis by ground-based instruments, streamers have been followed out to about $4.5 \mathrm{R}_{\odot}$ with balloon-borne instrumentation (Newkirk and Bohlin 1965), and rocket-carried coronagraphs have observed the continuum corona to about $9 \mathrm{R}_{\odot}$ (Tousey 1965).

The necessity of carrying instrumentation aloft is brought about by the relatively high scattered radiance of the terrestrial atmosphere. As has been pointed out, a reasonable requisite for measurement of the solar corona is that the radiance of the corona be at least $10 \%$ that of the residual sky background radiance (Newkirk and Bohlin 1963). Thus the limit of ground-based instruments is on the order of $10^{-6} \mathrm{~B}_{\theta}$, where $\mathrm{B}_{\Theta}$ is the radiance of the mean solar disk. Upon achieving altitudes of 20-30 km through the use of balloons, this detection limit is reduced to approximately $10^{-9} \mathrm{~B}_{\odot}$. Rocket flights (and also satellites) are essentially above the atmosphere, and the scattering characteristics of the sky background are no longer the limiting factors governing the performance of instrumentation carried in them, although a similar problem of scattering due to particulate matter carried aloft by the vehicle must be considered (Tousey 1965).

In a11 of the above situations, aside from the limitations imposed by the atmospheric stray radiance, one must contend with the presence of 
unwanted radiation introduced by the instrument itself. Newkirk and Bohlin (1963) have summarized the analysis of Lyot as to the origin of such stray radiance introduced by the coronagraph. The sources are: (a) diffraction at the objective lens aperture, (b) a solar Image produced by multiple reflections at the objective lens surfaces, (c) macroscopic inhomogeneties in the lens material, (d) surface defects in the lens, and (e) lens body scattering.

The evolution of the coronagraph has centered about the photographic visible portion of the spectrum, and for the experiment in question, the extension of such an instrument for use in the infrared must next be considered. The infrared coronagraph could take advantage of the wavelength dependence of the scattered light in the telluric atmosphere -for example, the near-solar sky radiance at $2.2 \mu$ would be expected to be on the order of $6 \times 10^{-2}$ times less than that at $0.6 \mu$, if a wavelength dependence of scattered light of the form $\lambda^{-2.2}$ is assumed, as found by Newkirk and his collaborators from their stratospheric balloon measurements of the sky radiance (Eddy, 1961; Newkirk and Eddy 1964). Further, it would be expected that the influence of body and surface scattering on the stray light in the coronagraph would be somewhat reduced at the longer wavelengths, although no quantitative measurements have been performed on this phenomenon. On the other hand, the contribution due to diffracted light in the instrument would necessarily increase as the ratio a/ $\lambda$ decreased; here a is a characteristic diameter of the optical system. A coronagraph for use in the near infrared, at $2.2 \mu$, has been constructed and evaluated; this instrument was successfully flown on a balloonborne platform to the stratosphere $(28 \mathrm{~km})$ on 9 January 1967 . This section 
will describe the instrument and its properties.

The flight instrument was composed of an external occulting assembly that shielded the primary lens from direct photospheric radiation, and a rear ("Lyot") section, which was made up of the primary objective, field aperture and field lens, and Lyot objective lens with the detection assembly. Figure 1 presents a schematic diagram of the coronagraph. The instrument differed from its predecessors in one important respect; the optical system was axial, and the corona was scanned radially by rotation of the Lyot section about the center of the primary lens. There was internal occulting, but not by a disk; the solar image was blocked by the field aperture plate, behind which was mounted the field lens.

\section{Externa1 Occulting Assemb1y}

In order to achieve the low level of instrumental stray radiance necessary for successful observation of the faint outer solar corona, the primary lens must be blocked from direct photospheric radiation. Then the stray light entering the coronagraph proper is due to forward scattering from discontinuities in the shielding surface edge, and diffracted light from that edge. In principle, the former contribution may be made negligible, and the latter reduced appreciably through the technique of apodization.

There are currently two feasible approaches to the technique -- the use of a series of circular disks whose peripheries shield those of disks nearer the sun (Newkirk and Boh1in 1963); and the use of a toothed disk (Purcell and Koomen 1963; Tousey 1965), the boundary of which provides the necessary illumination gradient. Both approaches have merits; we have 
compared the system herein described with smooth circular disks, since the latter offer distinct ease of construction.

With this infrared coronagraph, it was decided to scan out into the corona on one side of the solar disk only; hence we were able to make use of multiple straight edges placed appropriately for apodization purposes. A substantial reduction in the diffracted flux reaching the primary lens, as compared with that present from a circular disk assembly, could then be realized.

To quantitatively compare the effect of the straight edge and the circular disk, we have measured the flux incident upon a $10 \mathrm{~mm}$ diameter quartz objective lens, due to diffraction from a single circular disk that subtended an angular diameter of 48 minutes of arc as seen from the primary lens, and also a straight edge which subtended on one side of the optic axis an angular radius of 24 minutes of arc, and an angular radius of 100 minutes of arc on the opposite side (see Figure 2). The straight edge height subtended a distance of 100 minutes of arc, and the simulated solar radiation was provided by a tungsten strip filament lamp and lens assembly, which subtended an angular diameter of 32 minutes of arc as seen by the occulting assembly. The initial measurements were made at an effective wavelength of $0.5 \mu$, and also $0.8 \mu$, employing an ITT FW118 photomultiplier tube and appropriate filters in a chamber whose air was electrostatically cleaned and circulated. The stray radiance due to particulate matter was thereby reduced to about $1 \times 10^{-7} \mathrm{~B}_{0}$. For the circular disk, it was found that the stray radiance was $B \sim 4.6 \times 10^{-4} B_{0}$, where $\mathrm{B}_{0}$ was the radiance due to the direct simulated sun. In the case of the abovementioned straight edge, $B \sim 3.7 \times 10^{-6} \mathrm{~B}_{\Theta^{\circ}}$. Therefore, a factor of 
about $8 \times 10^{-3}$ in radiance reduction is realized in comparing the straight edge with a "comparable" circular disk. The former measurement is in substantial agreement with the measurement of Newkirk and Bohlin (1963), made on a scaled down version of their flying coronagraph, at $\lambda=0.54 \mu$. In addition to the measurement with the single circular disk at $0.5 \mu$, given above, the stray radiance from the same disk was also measured at $0.8 \mu$; the increase in radiance relative to that of the simulated sun was found to be $1.58 \pm 0.03$, which implied an approximate $\lambda^{+1}$ dependence of the diffracted radiance. This wavelength dependence was verified for a circular disk assembly composed of three disks whose diameters were $1.496 \mathrm{~cm}$, $1.421 \mathrm{~cm}$, and $1.346 \mathrm{~cm}$, respectively, placed $8.07 \mathrm{~cm}$ apart with the leading (toward the source) disk $107.2 \mathrm{~cm}$ from the small objective lens. The inferred wavelength dependence confirmed that the contribution to the stray radiance due to light scattered by minute imperfections in the edges of the disks was small; such a contribution would be expected to show a decrease in relative intensity with increasing wavelength.

The magnitude of the measured radiance due to the multiple circular disk assembly was found to be $\sim 2.9 \times 10^{-5} \mathrm{~B}_{\odot}$, at $\lambda=0.5 \mu$, which represented a decrease in radiance relative to that due to a single circular disk of about $6.3 \times 10^{-2}$; i.e., the radiance was reduced by about a factor of sixteen. This may be compared with the factor of 13.5 found by Newkirk and Bohlin (1963) for their model system.

A small multiple straight edge assembly, which subtended on one side of the optic axis the same angular distances as the radii of the above circular disk assembly, was measured at $\lambda=0.5 \mu$, and the stray radiance found to be $2 \pm 1 \times 10^{-7} B_{\odot}$. Therefore, within the limits of experimental 
error, the factor of $8 \times 10^{-3}$ in radiance reduction fround when comparing the single straight edge with the single circular disk, was also appropriate for the comparison of the multiple straight edge and circular disk assemblies.

Because the intensity of diffracted light, in the shadow zone, due to a straight edge, is proportional to $\lambda^{+1}$ (Born and Wolf 1964), it would be expected that the external straight edge assembly would introduce roughly four times more diffracted light at $2.2 \mu$ than at $0.5 \mu$. The only measurement at the $2.2 \mu$ wavelength was performed on the actual flight external occulting assembly. Here the occultation was accomplished through the use of three knife edges, placed $398.6 \mathrm{~cm}, 368.6 \mathrm{~cm}$ and $338.6 \mathrm{~cm}$, respectively, from the primary lens. The three edges were $5.565 \mathrm{~cm}$, $5.286 \mathrm{~cm}$, and $5.007 \mathrm{~cm}$ from the optic axis of the system, and formed the geometric inner limit of the field of view of the instrument. The edges were about $30 \mathrm{~cm}$ high, and hence subtended about 300 minutes of arc, as seen from the primary lens; the sides opposite the knife edges subtended about the same angle, measured from the optic axis. The $2.2 \mu$ diffracted radiance, measured in a long clean assembly constructed at Palestine, Texas prior to flight, was found to be $1 \pm 0.5 \times 10^{-7} \mathrm{~B}_{\odot}$. The fact that the magnitude of the diffracted radiance observed was somewhat less than that predicted on the basis of the measurements on the scaled system, and a knowledge of the wavelength dependence of the diffraction phenomena, is undoubtedly due to the fact that the beam area of the solar simulation device in the clean tunnel was smaller than the area of the external assembly; the actual beam was placed so that it encompassed the knife edges, but the opposite side of the assembly was not illuminated. 
The diffracted light reaching the primary lens was highly nonuniform across the lens aperture; the intensity as a function of distance from the edge of the primary aperture, on the same side of the optic axis as the knife edges, was measured, using a slit $5 \mathrm{~mm}$ wide, at four points within the aperture, and the results are given in Figure 3 . It is seen that by masking the primary aperture to a distance of about $0.50 \mathrm{~cm}$ from the aperture edge, the diffracted flux was substantially reduced (by about $50 \%$, along with an attendant reduction in the coronal flux of about $12 \%)$

Therefore, on the basis of the above measurements and evaluation, it is concluded that the diffracted radiance in the flight coronagraph, originating at the multiple knife edge assembly, was somewhat less than $1 \times 10^{-6} \mathrm{~B}_{\odot}$.

\section{Lyot Coronagraph Assembly}

The Lyot section of the infrared coronagraph was comprised of the primary objective and aperture, field stop baffle and field lens, Lyot objective, and detection assembly. Even though the photospheric radiation had been reduced by a factor $\sim 10^{-6} \mathrm{~B}_{\odot}$ by the external occulting assembly, it was necessary to reduce this unwanted radiation by at least a factor $10^{-5} \mathrm{~B}_{\odot}$ in order to observe the outer solar corona. Such reduction is common with instruments designed for the visible portion of the spectrum; the infrared instrument had to be proven.

It was of the utmost importance that the primary lens of the coronagraph be of high quality, and as free from imperfections as possible, for otherwise the remaining elimination of the unwanted photospheric flux would 
be seriously hampered. It was required, therefore, that the material meet the general requirements as outlined by Newkirk and Bohlin (1963) for consideration as a coronagraph primary element. To their 1ist was added the item, for our requirement, that the primary have relatively high transmission in the $2.2 \mu$ spectral region. A logical candidate was quartz, since previous work indicated that it met the necessary requirements for visible light coronagraphs, and since the $2.2 \mu$ transmission of quartz of thickness $20 \mathrm{~mm}$ was relatively high, on the order of $85 \%$. This figure is based on the observed transmission of the so-called "wet" quartz; that is, quartz which contains relatively large amounts of water or $(\mathrm{OH})^{-}$, i.e., typically 300-500 ppm. "Dry" quartz is also available, in which the water- $(\mathrm{OH})^{-}$content has been reduced, the effect being to increase the transmission on the $2.2 \mu$ region to over $90 \%$ for a $20 \mathrm{~mm}$ thick plate. Relative merits of these two types of quartz as pertained to their usefulness as a coronagraph primary objective were examined. Lenses were made using several different types of quartz currently available from various manufacturers. The evaluation consisted of using the quartz as the primary lens of the coronagraph, and noting the combined diffracted and stray light as a function of the position angle of the coronagraph during a simulated coronal scan. No field lens to reduce the diffracted component was employed. The measurements were made in the same electrostatically cleaned environment previously described, with a simulated solar disk subtending 32 minutes of arc as seen by the primary lens. The lenses were a11 $3.60 \mathrm{~cm}$ in diameter, and had a focal length of $13.10 \mathrm{~cm}$ at $\lambda=0.8 \mu$. The ITT FW118 photomultiplier tube was used, and the calibration determined with a set of standard opals. These opals were made by combining opal glass and gelatin neutral density filters, and were calibrated in terms of the 
radiance of the simulated solar disk. They are ineffective at $2.2 \mu$, and evaporated metal films are necessary there. The results, expressed in terms of the radiance of the artificial solar disk, are presented in Figure 4. The curves are for single samples of the types noted; however, they are representative of all the selected samples studied. (By "selected", it is meant that each objective was ground and polished from a blank which had been inspected for low bubble and inclusion content.) Further, among several single types of quartz, lenses were ground and polished by more than one optical company in an attempt to assess the resultant finishes and scattering properties. The lens selected for the flight coronagraph was finished by the McMinn Optical Company of Alexandria, Virginia.

Also plotted in Figure 4 is a curve of the calculated diffracted intensity due to the extended source, based upon the results of Zanoni and Hill (1965). The expression for the radiance of the diffracted light, in terms of that of the mean solar disk, due to a circular aperture, is, to sufficient accuracy,

$$
I(r)=\frac{\lambda f}{2 \pi^{2} \text { a } R_{\odot} r\left(r^{2}-1\right)} \text {, }
$$

where $r=R / R_{\theta}$, with $R_{\theta}$ the radius of the geometrical solar image, $\lambda$ the wavelength of light used, $f$ the focal length of the lens, and a the aperture radius.

An examination of Figure 4 shows that beyond position angles corresponding to $r \sim 11$, scattered light from the lens dominated the 
diffracted component. It is also seen (and verified by the other samples) that the dry quartz (General Electric Type 106, and the Englehard Infrasil I) produce somewhat higher levels of scattered radiance than the wet varieties (the exception was Dynasil UV 1000, a wet type, which produced uniformly high radiances in each of the three samples tested). The higher scattered radiances from the dry samples could be predicted, for examination of the blanks clearly showed a larger number of small ( $\sim 100 \mu)$ diameter bubbles than in the wet samples. On the basis of these measurements of seven selected samples of the dry quartz, it is concluded that such quartz produces unsatisfactorily high scattered radiances to be useful as a primary objective material.

The stray radiance due to the final selected primary objective lens, made of Englehard Homosil quartz, was also measured at $2.2 \mu$ as a function of the coronagraph scan angle. These measurements were made with a lead sulfide detector, and calibration in terms of the radiance of the mean solar disk accomplished through the use of evaporated metal "neutral density" filters. A wavelength dependence of the lens scattering may be inferred from these measurements, if Equation (II-1) is used to account for the diffraction component of the measured stray radiance. For example, at $\lambda=0.8 \mu$, at $r=13.3$, the diffraction theory predicts a radiance of about $1.5 \times 10^{-7} \mathrm{~B}_{\odot}$, while we have measured a total stray radiance of $8.0 \times 10^{-7} \mathrm{~B}_{\odot}$. The net scattered radiance is then $6.5 \times 10^{-7} \mathrm{~B}_{\odot}$, and is to be compared with the net scattered radiance at $2.2 \mu$, found to be $1.7 \times 10^{-7} \mathrm{~B}_{\odot}$. If it further is assumed that the lens scattering follows a wavelength law of the form $\lambda^{-k}$, then we determine $k$ to be 1.3 . In summary, from the measurements made on the primary lens, and interpreted 
in the above manner, we have found that the scattered radiance obeys a wavelength power law $\lambda^{-k}$, where $k=1.3 \pm 0.2$.

In the light of the measurements presented herein, and on visible light coronagraphs, it is appropriate to comment here on the suggestion of Zanoni and Hill (1965), who present calculations on the diffraction from a circular aperture for a disk source, and also from a square aperture for a disk source. They found that along the diagonal of the square aperture the diffraction is reduced some three orders of magnitude under that due to the circular aperture, at $r=2$; while for $r=4$, the reduction is about four orders of magnitude. They suggested that "the use of a square aperture would reduce the diffracted light by four orders of magnitude below the skylight background $\left(\sim 1 \times 10^{-6_{B_{0}}}\right)$ at a good ground-based observatory for one-half the azimuthal field and distances greater than 400 secs of arc from the solar limb $(r=1.4) "$. It is readily seen that such reductions cannot be realized in practice, for at $r=2$, the scattered radiance due to the objective lens itself would be, at least, some two orders of magnitude greater than the predicted diffracted radiance along the diagonal of the square aperture. We have examined the effect of this apodization of the primary objective of the flight coronagraph at $0.8 \mu$, and have found that the use of a square aperture along a diagonal allowed the reduction of the net stray radiance (diffraction plus scattering) from $3.1 \times 10^{-6} \mathrm{~B}_{\odot}$ to $2.0 \times 10^{-6_{\mathrm{B}}}$, at an angle corresponding to $\mathrm{r}=1.5$.

There is also the stray radiance produced by the primary lens due to the solar image formed by multiple reflections in the objective; the image is formed about $1 / 6$ th the focal length of the lens from the lens, and the radiance produced in the coronagraph is approximated by 


$$
\frac{4 \beta^{2}\left(1-\beta^{2}\right)}{25 \pi} \quad\left(\frac{f \theta_{\theta}}{D}\right)^{2} B_{\theta},
$$

where $\beta$ is the reflection coeffictent of the quartz, $f$ is the focal length and $D$ the diameter of the objective, and $\theta_{\theta}$ the angular solar diameter. For the system in question the stray radiance is found to be $\sim 6 \times 10^{-8}{ }_{\mathrm{B}_{0}}$, which is negligible relative to the other contributors to the stray light in the coronagraph.

The remalnder of the Lyot section employed a field lens, of GE type 151 quartz, $2.54 \mathrm{~cm}$ in diameter and $6.89 \mathrm{~cm}$ focal length, which imaged the bright diffraction ring of the primary objective aperture on the periphery of the Lyot objective, whose diameter was $3.50 \mathrm{~cm}$ and focal length was $5.65 \mathrm{~cm}$. The detection assembly was used for both the eclipse and coronagraph observations, and will be described in the next section.

The stray radiance present in the complete Lyot section was measured in the clean environment for $\lambda=2.2 \mu$, as a function of the coronagraph scan angle, and was found to be $2.7 \times 10^{-6} \mathrm{~B}_{\Theta}$ at an angle corresponding to $r=3.7$. This stray radiance decreased monotonically to a level of $7.0 \times 10^{-7} \mathrm{~B}_{0}$ at $\mathrm{r}=16$, approximately the outer scan limit.

\section{Conclusion}

If it is assumed that the net stray radiance in the complete flight coronagraph assembly is the product of the measured radiances for each of the two separate components, this net radiance is less than $3 \times 10^{-12} \mathrm{~B}_{0}$ at the inner scan limft, and decreases monotonically as the scan angle is increased. The actual measurement of the net stray radiance in the 
complete flight coronagraph was not made, and would require a large evacuated chamber in which to conduct the test in order to reduce the residual radiance of the air column present, together with a source several orders of magnitude brighter at $2.2 \mu$ than the tungsten strip filament lamps used in this work. 


\section{INSTRUMENTATION AND CALIBRATIONS}

\section{Eclipse Instrumentation}

The ground-based $f / 3.1$ telescope employed a $29.2 \mathrm{~cm}$ diameter spherical mirror, of focal length $91.4 \mathrm{~cm}$ (see Figure 5). A newtonian pick-off mirror directed the beam to a dry lce refrigerated chamber, the same chamber used with the infrared coronagraph. Housed in the chamber were the tuning fork chopper, spectral bandpass filter, and the lead sulfide detector, the area of which was $2.31 \times 10^{-2} \mathrm{~cm}^{2}$. The field of view of the telescope was $0.108^{\circ}=0.406 R_{\text {o }}$ square. A system external to the main telescope tube allowed the focussing of radiation on the detector from a blackbody source of nearly unity emissivity and variable controlled temperature for radiometric calibration.

The spectral bandpass filter was of the wide bandpass type, with average transmission of $82 \%$ over the wavelength range $2.02-2.45 \mu$. Effectively zero transmission, i.e., transmission less than $10^{-4}$, was reached on the short wavelength side at $1.96 \mu$, and on the long side at

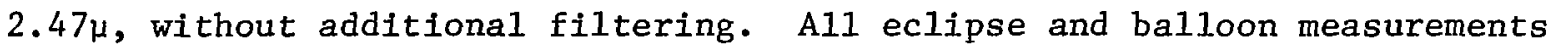
were made with the above spectral bandpass; we have designated their effective wavelength as $2.2 \mu$.

The electronic system was standard, employing synchronous rectification with the Princeton Applied Research Corporation Model HR-8 system. The d.c. output was displayed on a chart recorder in five steps of sensitivity -- $1,5,25,126$, and 250 , relative to the input. Also recorded were time pulses each second, and the temperature of the lead sulfide detector through the use of a bead thermistor inside the refrigerated chamber. 
Aligned with the telescope was an $8 x$ viewing telescope with illuminated reticle system, and a $35 \mathrm{~mm}$ cine camera with a $210 \mathrm{~mm}$ focal leng.th lens, with a similar reticle system. The latter was to be used for positional information during totality; however, it jammed 17 seconds before second contact and was useless. Positions during totality were noted on recording tape with a telephone system. We estimate positional errors of $\pm 0.2 R_{0}$ in all scans during totality, the relatively large uncertainty arising because of the loss of the cine system.

The responsivity of the detector system was measured in the laboratory, using blackbody reference sources, and found to be $1.5 \times 10^{5}$ volts/watt, with a noise equivalent power of $2.2 \times 10^{-13}$ watts. The NEP measurements were made with a $500{ }^{\circ} \mathrm{K}$ blackbody source, 400 cps chopping, and an electrical bandwidth of $0.016 \mathrm{cps}$. If the radiance of the mean solar disk is taken to be (Allen 1963) $B_{\theta}=105$ watts $/ \mathrm{cm}^{2}$-ster- $\mu$, the above NEP corresponds to a radiance of $1.4 \times 10^{-11} \mathrm{~B}_{\mathrm{e}}$. Responsivity and NEP measurements were repeated at the eclipse site, with essentially the same results; however, several minutes before totality the noise level of the system increased to approximately eight times the previously measured value, possibly due to the malfunction of an ancillary experiment, and remained constant throughout the observation period. The NEP during the observations was $1.1 \times 10^{-10} \mathrm{~B}_{\odot}$, and we have analyzed no recorded values of the coronal radiance whose signal: noise ratio was less than $2: 1$; as will be seen, this corresponded to the measured coronal radiance at about $6 R_{\odot}$. Pointing of the telescope was achieved by two servo-motors, controlled by the observer, driving the telescope in and perpendicular to the plane of the ecliptic, the position of which calculated for the time of eclipse. 
During totality, two scans from $2 \mathrm{R}_{0}$ to $10 \mathrm{R}_{0}$ in the ecliptic were made, together with an observation of the corona at $5 R_{\text {e }}$ perpendicular to the ecliptic plane.

Mounted along the main telescope tube was also a photometer, boresighted with the Infrared system, the field of view of which matched that of the infrared system. The photometer objective was an Jaegers achromat: $5 \mathrm{~cm}$ diameter and $76 \mathrm{~cm}$ focal length, and a filter selected a bandpass of $385 \mathrm{~A}$ at $5600 \mathrm{~A}$. The detector was an EMI $9524 \mathrm{~S}$ end-on photomultiplier with a sensitivity of 2000 a/lumen at 1700 volts applied potential. The d.c. output fed an operational amplifier and then the chart recorder, through attenuation steps of $1,2,5,50$, and 300 .

With the photometer system, it was hoped to record the radiance of the corona in the visible, averaging over the same spatial region as with the infrared system. Failure of the high voltage system prior to totality, probably due to arc-over because of dust, vitiated any information from the system.

\section{Eclipse Calibrations}

Radiometric calibration of the system (exclusive of the main telescope) was accomplished with the blackbody reference source and thin-film neutral density filters. Such a calibration resulted in an experimental mean solar radiance, corrected to zenith, of $B_{e}=90$ watts $/ \mathrm{cm}^{2}-\mathrm{ster}-\mu$, where a calculated spectral throughput of the system of $1.47 \times 10^{-3} \mathrm{~cm}^{2}$-ster- $\mu$ has been used. The above value is about $14 \%$ lower than the value quoted by Allen (1963). Direct calibration on the solar disk was performed before and after totality by the use of a mask, placed over the main tube, with 
three circular holes of diameter each $3.18 \mathrm{~mm}$ covered with a thin-film neutral density filter. The net attenuation of this assembly was $8.9 \times 10^{-8}$, and the resultant signals were convenient to compare with the coronal deflections. Thermal emission from the assembly was approximately $1 \times 10^{-9} B_{e}$, or about $1 \%$ of the total flux incident on the detector. The above calibrations necessarily depend upon the linearity of the detection device and electronic system; the linearity of our system was examined over ranges of incident power from $3 \times 10^{-7}$ watts to the noise equivalent power of about $2 \times 10^{-13}$ watts, and no devlations from linearity exceeding $\pm 5 \%$ were found. All signal levels recorded, both during eclipse, and during the balloon observations, fell within the above range.

\section{Telescope Instrumental Stray Light}

When attempting to observe a phenomenon such as the solar corona which shows rapid radiance changes over relatively small spatial regions, it is necessary to examine the instrumental influence of diffracted and stray light. For this reason the stray light in the eclipse instrument has been examined, using as a source a tungsten strip filament lamp and a quartz objective lens which subtended an angle of $32^{\prime}$ as seen by the telescope. This solar simulator could then be positioned to examine the radiance of stray light as a function of radial distance from the solar center. Figure 6 presents the results of such measurements, together with a curve of the calculated diffracted intensity, for such an extended source. It is seen that the instrumental stray light contributes negligibly to the coronal measurements, since the flux incident upon the detector is only 
$1 \times 10^{-3}$ that due to the source, when the telescope was pointed to within 0.23R. ( $\left.\sim 4^{\prime}\right)$ of the simulated solar disk. If it is assumed that the optics were of equivalent cleanliness in the field, the coronal measurements were not affected by stray light. Conditions on site were not favorable in this respect; however, the primary and newtonian mirrors were cleaned in situ carefully one hour before totality and the main tube kept closed.

\section{Eclipse Sky Background and Zenith Corrections}

There are no published measurements of the sky background in the infrared during eclipse. Blackwel1 (1952) assumed zero sky background at $1.9 \mu$ during the eclipse of 25 February 1952, based on his daylight sky measurements and measurements on the night sky near full moon. Various measurements in the visible region of the spectrum have been performed, and a reasonable mean value is approximately $2 \times 10^{-9} \mathrm{~B}$ at $6000 \mathrm{~A}$. Blackwel1 (1955) assumed a Rayleigh sky and found that for altitudes up to about 30,000 feet, the brightness of the eclipse sky varied approximately as the atmospheric pressure, although the sky background measured from an aircraft during the eclipse of $20 \mathrm{July} 1963$ by Blackwell and Petford (1966) was three times darker than that measured under similar conditions in 1954. Further, Newkirk and Bohlin (1963) found that near the sun the wavelength dependence is given by $\lambda^{-2.2}$, rather than by the Rayleigh law. The sky brightness for the site, then, was approximately.

$$
\begin{aligned}
B_{S} & \sim(0.6 / 2.2)^{2.2}(18.4 / 29.9)\left(2 \times 10^{-9} B_{\odot}\right) \\
& =7.2 \times 10^{-11_{B_{\theta}}}
\end{aligned}
$$


which, because of the increased noise level of our system during totality, was undetectable. It was assumed, hence, that the background during totality was negligible.

To correct all measurements to zenith, the data obtained on direct photospheric calibrations were fitted by a curve of the form $1+k \sec \theta$, where $\theta$ is the solar zenith angle and $k$ is an extinction coefficient, including absorption and scattering, determined from the fit. In addition to the two photospheric calibrations made on eclipse morning, similar data obtained on site October 31 , November 3,8 , and 9 , when sky conditions were good, were used. Best fit of the data occurred for $k=0.09$, and the zenith correction factor for the eclipse data was hence $1+0.09$ (1.269) $=1.11$.

\section{Local Circumstances}

Some details of the site used by the joint High Altitude ObservatoryJohns Hopkins University expedition, near Pulacayo, Bolivia, have been published. (Malville 1967) The latitude was $20^{\circ} 14.8^{\prime} \pm I^{\prime}$ S and longitude $66^{\circ} 32.5^{\prime} \pm 0.5^{\prime} \mathrm{W}$, determined by $\mathrm{W}$. Hatt of the High Altitude Observatory. This placed our observation point approximately four miles north of the eclipse center line, and the estimated duration of totality was 93 seconds. Second contact occurred approximately at $13^{\mathrm{h}} 25^{\mathrm{m}} \mathrm{U} . \mathrm{T}$., and the solar zenith angle was $38^{\circ}$. Just prior to totality the near-solar sky as seen from our 13,000 feet altitude was exceptionally clear, but soon after totality cirrus clouds became noticeable. 


\section{Ba11oon Instrumentation}

The design of the optical train of the coronagraph was discussed in Section II; the entire instrument was mounted on the trunnion of the platform used by Laboratory of Astrophysics and Physical Meteorology in the past balloon astronomy program (cf. Strong 1967).

The limits of radial pointing for the Lyot section were $2.7 R_{\theta}$ and $16.2 R_{0}$, and the rate of scanning was $1.25 R_{0}$ per minute, found to be linear to within $\pm 1 \%$ over the above range. Solar tracking was accomplished through the coarse tracking system as described by Bottema (1967). The azimuth error deadband during the period of observations, from 15:00 to 20:15 U.T., remained constant at \pm 5.5 minutes of arc, while the elevation error remained less than \pm 1 minute of arc. The oscillation period was also constant and approximately 20 secs. Because of these oscillations across the deadband, the scans of the corona were interrupted by discontinuities each $0.21 \mathrm{R}_{\rho}$, when the field of view moves $0.69 \mathrm{R}_{0}$ ( 11 minutes of arc). Superposed on the azimuth tracking error was a small oscillation of magnitude \pm 1 minute of arc, present throughout the flight. Anticipating the results of the observations, the effect of such an oscillation on the continuum corona observed may be assessed. If it is assumed that the "true" coronal radiance is of the form

$$
\mathrm{B}_{\mathrm{c}}(\text { true }) \propto \mathrm{R}^{-\mathrm{k}}
$$

then it can be shown that the measured radiance is given, to a first approximation, by 


$$
B_{c}(\text { measured })=B_{c}(\text { true })\left[1+\frac{k(k+1)}{2} \frac{(\Delta R)^{2}}{R_{0}}\right] \text {, }
$$

where $\Delta R$ is the movement of oscillation, about the position $R_{0}$. We have assumed the period of oscillation is small with respect to the actual detection time constant used. The measured continuum radiance varies approximately as $R^{-5}$. At $3.7 R_{\odot}$, then, the measured radiance exceeds the actual value by only $0.5 \%$. Of course, at larger distances from the solar limb, the effect is still smaller. Simultaneous with the data recording was a recording of the output of sensor eyes aligned with the solar disk, so that a complete record of the behavior of the tracking system was obtained, and reduction of the coronal radiance as a function of radial position was possible. Maximum errors in position are estimated to be $\pm 0.12 R_{e}$, or about 2 minutes of arc, with this error due primarily to possible misalignment of the tracking eyes with the optical axis of the coronagraph.

For the experiment in question the optical train of the Lyot section was required to be relatively fast; this meant that the focal plane of the external knife edges would be considerably displaced from that of the coronal image. In fact, the actual displacement of $0.600 \mathrm{~cm}$ lead to a circle of confusion whose radius was $0.077 \mathrm{~cm}$, or $1.18 \mathrm{R}_{\theta}$, where $R_{\theta}$ was the size of the solar radius in the coronal focal plane. The field of view of the system was 7.4 minutes of arc wide (and 24 minutes of arc high), so that the field limiting aperture moved out of the influence of the edges of the apodizing elements when pointed about 74 minutes of arc from the optic axis. The inner limit of the field of view could be adjusted by movement of the position of the image of the actual solar disk through 
positioning of the solar tracking eyes. For the balloon flight the eyes were positioned so as to move the solar Image $20 \pm 2$ minutes of arc; the effective inner limlt of the field was then about $3.6 R_{\odot}$.

Also recorded during flight were the system voltage and the detector temperature, through a thermistor sensor imbedded on the detector flake mounting block inside the refrigerated chamber. Over the duration of the flight the coolant, solid $\mathrm{CO}_{2}$, gradually dissipated and the temperature of the flake rose. The temperature range that occurred was $-109^{\circ} \mathrm{C}$ to $-100^{\circ} \mathrm{C}$ during the 5.25 hours of observations. A correction for the resultant decrease in sensitivity of the detector has been employed, with a maximum correction of $13 \%$.

The synchronous rectifier amplifier system used in the eclipse work was also employed for the balloon system. The unit, a Princeton Applied Research Corporation Model HR-8, was contained in a tank sealed to pressure changes from the ground to altitude. The tank was thermally insulated, and the interior temperature recorded. As will be seen, the sky radiance, corrected to zenith, shows variations throughout the day. The possibility that these variations are in actuality due to drifts in the d.c. output of the amplification system cannot be excluded. However, the fact that the measured temperature in the pressure tank increased smoothly throughout the flight lends strong weight to the possibility that such sky radiance changes are indeed real. Finally, from measurements made on the d.c. voltage drift with temperature of the electronic system prior to flight, a maximum change (in terms of the mean solar radiance) of about $1 \times 10^{-10_{B}}$, or about $20 \%$ the measured radiance of the sky, could be expected. 


\section{Balloon Flight Calibration}

During the balloon flight, a scatter plate was employed to record signals from direct photospheric radiation. This was done for periods of approximately 5 minutes each, three times during float. Scattered photospheric radiation from the aluminum plate was reflected from a small mirror surface mounted to a shutter mechanism, actuated by command from an on-board programmer, and thence passed through the coronagraph proper. Ground measurements, using a series of thin-film neutral density filters, indicated the above system provided an attenuation by a factor of $6.0 \times 10^{-9}$. Since the Lyot section continued to rotate during the period of calibration, the angle of incidence on the diffusing plate was altered as much as $4^{\circ}$. Ground measurements of the attenuation of the system as a function of this angular change were made; however, during the actual flight all three calibration periods had common angles, and any such angular effect can be eliminated. A liberal estimate of the blackbody radiation from the calibration assembly leads to a value $8 \times 10^{-11_{B}}$, which was negligible relative to the scattered photospheric radiation allowed to enter the coronagraph during the calibration periods.

\section{Coronagraph Instrumental Stray Light}

Evaluation of the coronagraph appeared in Section II. Measurements were made on the intensity and distribution of the stray light in the system, employing a solar simulation device, in an electrostatically cleaned environment. The results of these measurements indicated that beyond position angles corresponding to $3.7 \mathrm{R}_{e}$, the stray light in the instrument nowhere exceeded a radiance corresponding to $3 \times 10^{-12} \mathrm{~B}_{\Theta}$, and was a smoothly 
decreasing function of position angle. As will be seen, such a radiance was negligible relative to that of the corona and sky background, and cannot be responsible for the features observed on the continuum corona.

\section{Stratospheric Sky Background}

For the stratospheric platform observations, the smallest coronal signals that can be observed are determined by the magnitude and temporal variation of the various background radiations. These generally can be classed as emission and scattering processes, and included in the former category are both blackbody atmospheric emission and radical emission. An estimate of the former, using an emissivity of 0.05 for the sky at $28 \mathrm{~km}$, leads to a thermal radiation radiance of the sky of

$$
\mathrm{B}_{\mathrm{s}}(\text { therma1 emission }) \sim 1 \times 10^{-14} \mathrm{~B}_{\odot},
$$

for $2.2 \mu$, which is completely negligible in this experiment. Insofar as emission from radicals is concerned, the chief contributor to the sky background would be the $\mathrm{OH}^{-}$radical. In the wavelength region investigated (1.97-2.47), we expect contribution from the 8-6 and $9-7$ vibrational transitions; their intensity has been calculated (Chamberlain 1961), and leads to a radiance of about

$$
\mathrm{B}_{\mathrm{S}}\left(\mathrm{OH}^{-} \text {emission }\right) \sim 8 \times 10^{-12} \mathrm{~B}_{\mathrm{e}}
$$

Noxon and Vallance Jones (1961) observed the night sky in the $2 \mu$ spectral region; they place an upper limit of $2 \times 10^{-10_{B}}$ on any feature between 2.3-2.7 $\mu$, a value which is about twenty-five times the predicted $\mathrm{OH}^{-}$emission above. 
Newkirk and Eddy (1964) have made a thorough study of the daytime sky radiance in the visible region of the spectrum, and from the parameters deduced from their study, Newkirk and Bohlin (1963) presented calculated radiances for various near infrared wavelengths, and predicted the form of the stratospheric solar aureole for these wavelengths. Their estimate of the sky radiance at $2.2 \mu$ was, for an altitude of $25 \mathrm{~km}$,

$$
\mathrm{B}_{\mathrm{s}}(\text { scattering }) \sim 1.8 \times 10^{-9} \mathrm{~B}_{\mathrm{O}} \text {, }
$$

and the predicted aureole was essentially constant in radiance to about $9 R_{0}$, where it begins to fall off slightly (a total radiance change of about $2 \%$ at $\left.12 R_{0}\right)$. As mentioned, position angles corresponding to $3.7 R_{\ominus}$ to an outer limit of $16.2 \mathrm{R}_{\text {e }}$ were scanned. The coronal contribution to the resultant signal was sensibly zero beyond $10 \mathrm{R}_{\odot}$, so that essentially the radiance of the stratospheric sky over position angles from $2.93^{\circ}$ ( $11 R_{\vartheta}$ ) to $4.32^{\circ}\left(16.2 R_{\odot}\right)$, at intervals throughout the observation period, was measured. Figure 7 presents this sky radiance, in terms of the mean solar radiance, as a function of time. The curve has been corrected to zenith, in a manner described below. Two points concerning the measured sky radiance are noted: First, the non-uniform behavior of the sky radiance throughout the observing period, especially in the period from 17:30 U.T. to $19: 10 \mathrm{U} . \mathrm{T}$. It has been previously stated that it is believed such behavior is real; one possible explanation is that during the period of observation the balloon package floated over increasingly heavy terrestrial cloud cover and the higher radiance later in the day reflects the contribution due to backscatter from the cloud deck. Indeed, such a situation relative to cloud cover was observed to prevail, but it is difficult to 
assess the magnitude of such an effect. The most serious error in the observed coronal radiance would arise during a coronal scan while the sky radiance was changing. The following criterion for the data has been established: if the change in sky radiance during a scan exceeded $10 \%$ of the observed coronal radiance at $10 \mathrm{R}_{\odot}$, $1 . \mathrm{e}$, exceeded $6 \times 10^{-12} \mathrm{~B}_{\odot}$, then that scan has been refected. This has led to the rejection of nine scans throughout the day, and there remain eighteen that satisfy the criterion. Of these, thirteen scans were made during periods when the sky radiance change was $\lesssim 3 \times 10^{-12} \mathrm{~B}_{\odot^{\circ}}$ Second, in order to measure accurately the coronal radiance contribution to the measured signal, the sky radiance must be extrapolated inward toward the solar limb. The process is simplified because of the fact that the aureole is quite flat over the angular range in question -- about $3^{\circ}$ inward. This point is discussed elsewhere (MacQueen 1967) but from the aureole radiance measurement over angular range $2.9^{\circ}$ to $4.3^{\circ}$, a radiance change from $3^{\circ}$ inward of less than $2 \%$ has been Inferred.

\section{Zenith Correction}

As mentioned, the scattered radiation from photospheric disk was observed three times during float, and the observed deflections have been fitted to a curve of the form $C(1+k \sec \theta)$, where $\theta$ is the solar zenith angle, $k$ an extinction coefficient, and $\mathrm{C}$ a detector efficiency factor, whose use is made necessary due to the fact that the detector flake temperature changed during the period of observation. C ranges from 1.00 at $15: 00 \mathrm{U.T}$. to 1.13 at the completion of the flight, and best fit for the above arises for $k=0.05$. This extinction coefficient is in 
agreement with that which may be inferred from the data of Kondratiev, et al. (1967). The sky radiance measurements, of course, have been adjusted to zenith by a factor $\mathrm{C} \sec \theta$. All measurements presented have been corrected to zenith. 


\section{RESULTS AND DISCUSSION}

The data obtained from the period of totality of eclipse, and from the balloon observations over a period of 5.25 hours, allow a "picture" of the $2.2 \mu$ infrared corona to be formed over the position angles from $20^{\circ}$ north to $40^{\circ}$ south of the ecliptic and from $3.7 \mathrm{R}_{\odot}$ to $10 \mathrm{R}$ (from the balloon observations) and also in the ecliptic, from $2 \mathrm{R}_{\odot}$ to $6 \mathrm{R}_{\odot}$, and a single point perpendicular to the ecliptic at $5 R_{\odot}$ (from the eclipse). We now examine the results from both experiments in some detail.

\section{Eclipse Observations}

Figure 8 presents partial results of the two scans of the corona in the ecliptic plane, from the east limb of the sun. The two features superposed on the continuum coronal radiance are centered at about $3.5 R_{0}$ and $4 R_{0}$, respectively. The latter, more prominent, feature rises above the continuum background to a magnitude of radiance $\sim 9 \times 10^{-10_{\mathrm{B}}}$, while the former feature is of approximate radiance $1 \times 10^{-10_{\mathrm{B}}}$. It is believed that these features are due to the vaporization of solid material near the sun. Peterson (1967), observing at a site nearby, but with rather poor sky conditions, has made similar measurements, and has also detected the presence of the feature at $4 \mathrm{R}_{0^{\circ}}$. His data is presented in terms of an absolute calibration, and he found the magnitude of the feature at $4 R_{0}$ to be $2 \times 10^{-7}$ watts/ $\mathrm{cm}^{2}-s t e r-\mu$, which, if $\Lambda 1$ len's value for the solar radiance at $2.2 \mu$ is assumed, leads to a radiance of $2 \times 10^{-9} \mathrm{~B}_{0}$, which is somewhat more than a factor of two greater than the value found herein. Further, there is considerable difference between the measurements of the 
continuum corona -- at $5 R_{0}$ Peterson finds $2.9 \times 10^{-7}$ - watts $/ \mathrm{cm}^{2}-$ ster- $\mu$, or $3 \times 10^{-9} \mathrm{~B}_{0}$, while this experiment found the coronal radiance at the same distance, in the ecliptic, to be $4.4 \times 10^{-10_{B}}$. These differences are doubtless due to the sky conditions; Peterson's observations were made through light cirrus clouds. Peterson has also seen the feature at $4 R_{0}$ outside of eclipse, at a different site, under exceptional sky conditions, and has assigned a magnitude of $3.1 \times 10^{-7}$ watts $/ \mathrm{cm}^{2}$-ster- $\mu$ for the feature,

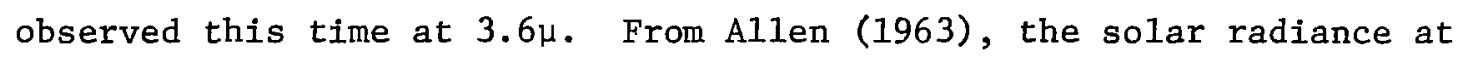
$3.6 \mu$ is 20 watts $/ \mathrm{cm}^{2}-s t e r-\mu$, so his measurement outside of eclipse at the longer wavelength yields a radiance of $1.5 \times 10^{-8} \mathrm{~B}_{0}$.

Finally, our single measurement made at $5 \mathrm{R}_{\odot}$ in a plane perpendicular to the ecliptic, leads to a continuum radiance of about $2.4 \pm 0.5 \times 10^{-10_{B}}$.

\section{Balloon Observations}

Thermal features, superposed on the continuum corona, were also observed during the balloon flight, and are shown in Figures 9 and 10 . These figures are composite curves, including the contributions of all eighteen useable scans. The observed radiance of the continuum corona is given in column 1 of Table I. The systematic errors of calibration are estimated to be on the order of $\pm 20 \%$, and may be due to the presence of stray light in the coronagraph during the ground calibration procedure, warpage of the optical train during the flight, and the possible departure of alignment of the calibration system during the flight, because of differential cooling.

The observed features have the following radiances, over and above that of the continuum coronal radiance; for the feature at $4 R_{e}$, the magnitude 
is about $1.2 \times 10^{-9} \mathrm{~B}_{\odot}$; for the features at $8.7 \mathrm{R}_{\odot}$ and $9.2 \mathrm{R}_{\odot}$, the radiances are about $3 \times 10^{-11} \mathrm{~B}_{0}$, and $4.2 \times 10^{-11} \mathrm{~B}_{\bullet}$, respectively. The observed radiance of the feature at $4 R$, is to be compared with that obtained during the eclipse (about $9 \times 10^{-10_{B_{\odot}}}$ ). The lower radiance of the peak observed during the eclipse is due to the relatively high scan speed used then; the shape of the feature is also seen to be somewhat altered. Further, it is noted that the continuum coronal radiance observed during the eclipse and balloon flight overlap in the region $3.7 R_{0}$ to $6 R_{\odot}$; over this region they agree to $5-7 \%$ in magnitude (the comparison is made with the ecliptic scan of the balloon flight). The signal:noise ratio of the balloon data over this range is, of course, much superior to that obtained with the eclipse instrument.

Figure 11 presents isophotes obtained from the continuum corona during the balloon observation period. The data plotted were obtained over periods of about 2.4 minutes (the time of scan from about $5 R_{\odot}$ to $8 R_{\odot}$ ) at intervals throughout the day. Of special interest is the increase in radiance near the ecliptic. The rate of rotation of the ecliptic during the period of interest was sensibly constant, and equal to $0.2^{\circ} /$ minute. During each period of observation from which radiances for the isophotes have been used, then, the ecliptic moved approximately $0.5^{\circ}$. It is seen that the interpolated "peak" of the isophotes would lie $1^{\circ}-2^{\circ}$ south of the ecliptic plane, and, in fact, coincides closely with the invariable plane of the solar system, which is about $1.4^{\circ}$ south of the ecliptic line. 


\section{$\underline{\text { Conclusion }}$}

The observations have provided the first picture of the outer solar corona in the near infrared, and as such, have stimulated basic questions concerning the corona and the interplanetary medium. Observation of the various features superposed on the background corona sheds some light on the question of the ultimate fate of the interplanetary dust, and also indicates that the oft-ressurected arguments for a major heliocentric contribution to the observed coronal radiance must be revised. The actual plane of symmetry of the zodiacal light is a questinn basic to the understanding of its origin; these observations seem unambiguously point to the invariable plane as the symmetry axis.

A more detailed treatment of the implications of the data presented herein may be found elsewhere (MacQueen 1967b).

Finally, more accurate observations, with higher spatial resolution and at several infrared wavelengths, will allow better specification of the parameters of the thermal component of the corona, and act as a greater stimulus to theoretical considerations of the interplanetary material and medium. 


\section{BIBLIOGRAPHY}

Anderson, W. H. 1924 Z. f. Phys. 28, 289.

Belton, M. J. S. 1967 paper presented at I.A.U. Symposium on Zodiacal Light and Interplanetary Medium, Honolulu, Hawaif.

Blackwe11, D. E. 1952 M.N. 112, 652.

Blackwe11, D. E. 1955 M.N. 115, 629.

Blackwel1, D. E. and Petford, A. D. 1966 M.N. 131, 383.

Born, M. and Wolf, E. Principles of Optics, New York, Macmillan Company. Bottema, M. 1967 App1. Opt. 6, 213.

Eddy, J. A. 1961 Thesis, University of Colorado, Boulder.

Harwit, M. 1964 Mem. Soc. Roy. Sci. Leige |5| 9, 506.

Ingham, M. F. 1961 M.N. 122, 157.

Kaiser, C. B. 1967a paper presented at I.A.U. Symposium on Zodiacal Light and Interplanetary Medium, Honolulu, Hawaii.

Kaiser, C. B. $1967 \mathrm{~b}$ paper presented at 124th meeting of American Astronomical Society, Williams Bay, Wisconsin.

Kondratiev, K. Ya., Nicolsky, G. A., Badinov, I. Ya., and Andreev, S. D. 1967 App1. Opt. 6, 197.

Lazarev, A. I. 1964 Soviet Astron.-A.J. 8, 285.

Low, F. J. and Smith, B. J. 1966 Nature 212, 675.

Lyot, B. 1930 C. R. Acad. Sci. Paris 191, 834 .

Lyot, B. 1931 ibid. 193, 1169.

Lyot, B. 1939 M.N. 99, 580.

MacQueen, R. 1967a J. Atm. Sci. (in press).

MacQueen, R. 1967b Dissertation, The Johns Hopkins University, Baltimore, Md. 
Malville, J. M. 1967 Sky and Telescope 33, 136.

Mangus, J. and Stockhausen, R. 1966 NASA Goddard Space Flight Center

Report X-614-66-29.

Moroz, V. I. and Boiko, P. N. 1965 Soviet Astron.-A.J. 9, 89.

Newkirk, G. A. and Bohlin, J. D. 1963 App1. Opt. 2, 131.

Newkirk, G. A. and Bohlin, J. D. 1965 Ann. Astrophys. 28, 234.

Newkirk, G. A. and Eddy, J. A. 1964 J. Atm. Sci. 21, 35.

Over, J. 1958 Kon.Ned. Akad. Weten. Proc. 51B, 74.

Peterson, A. W. 1963 Ap. J. 138, 1218.

Peterson, A. W. 1964 Ann. N. Y. Acad. Sci. 119, 72.

Peterson, A. W. 1965 private communication.

Peterson, A. W. 1967 Ap. J. 148, L37.

Purce11, J. D. and Koomen, M. J. 1962 paper presented at the spring meeting, Optical Society of America, Washington.

Russe11, H. N. 1929 Ap. J. 69, 49.

Strong, J. 1967 Appl. Opt. $\underline{6}, 179$.

Taylor, J. H. and MacQueen, R. M. 1964 Appl. Opt. 3, 1365.

Tousey, R. 1965 Proceedings I.A.U. Symposium No. 23, p. 293. 
TABLE I

Separation of the Observed Infrared Corona

\begin{tabular}{|c|c|c|c|}
\hline$R_{0}$ & Experimental & "Mean" K-corona & $\begin{array}{l}\text { Residual Continuum } \\
\text { Corona }\end{array}$ \\
\hline 3 & 45.0 & 15.5 & 29.5 \\
\hline 4 & 11.3 & 3.90 & 7.4 \\
\hline 5 & 4.20 & 1.25 & 2.95 \\
\hline 6 & 2.45 & 0.68 & 1.80 \\
\hline 7 & $1.67 \pm 0.05$ & 0.40 & 1.27 \\
\hline 8 & $1.15 \pm 0.07$ & 0.25 & 0.90 \\
\hline 9 & $0.78 \pm 0.15$ & 0.16 & 0.62 \\
\hline 10 & $0.5 \pm 0.2$ & 0.08 & 0.4 \\
\hline
\end{tabular}

Note: Al1 radiances are given in terms of $10^{-10}$ the radiance of the mean solar disk at $2.2 \mu$; errors given reflect uncertainties in the measurement of the actual deflections, and not possible systematic errors of calibration. 

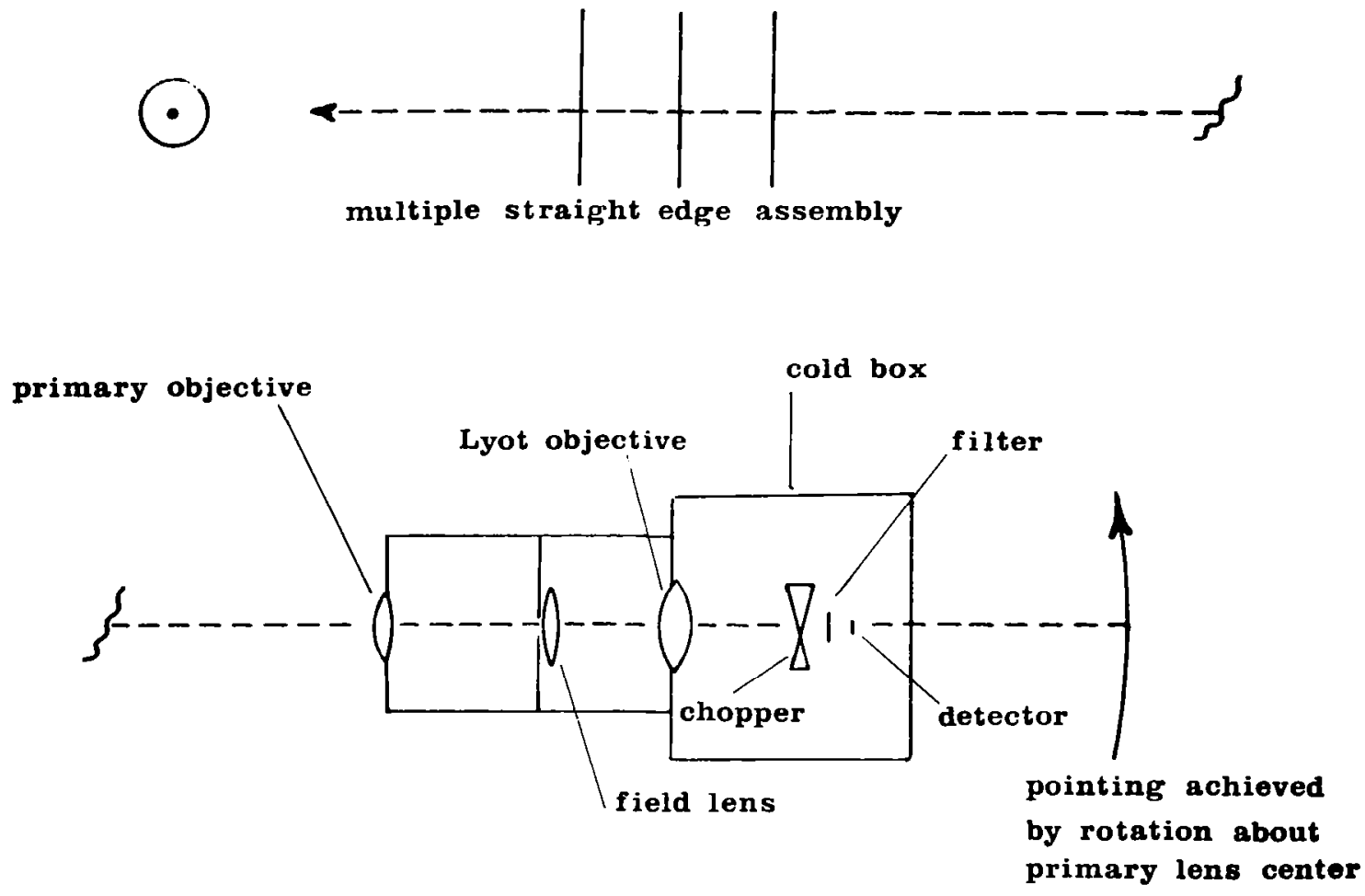

Figure 1.- Schematic representation of the balloon borne infrared coronagraph. The front knife edge was placed $398.6 \mathrm{~cm}$ from the primary objective. 


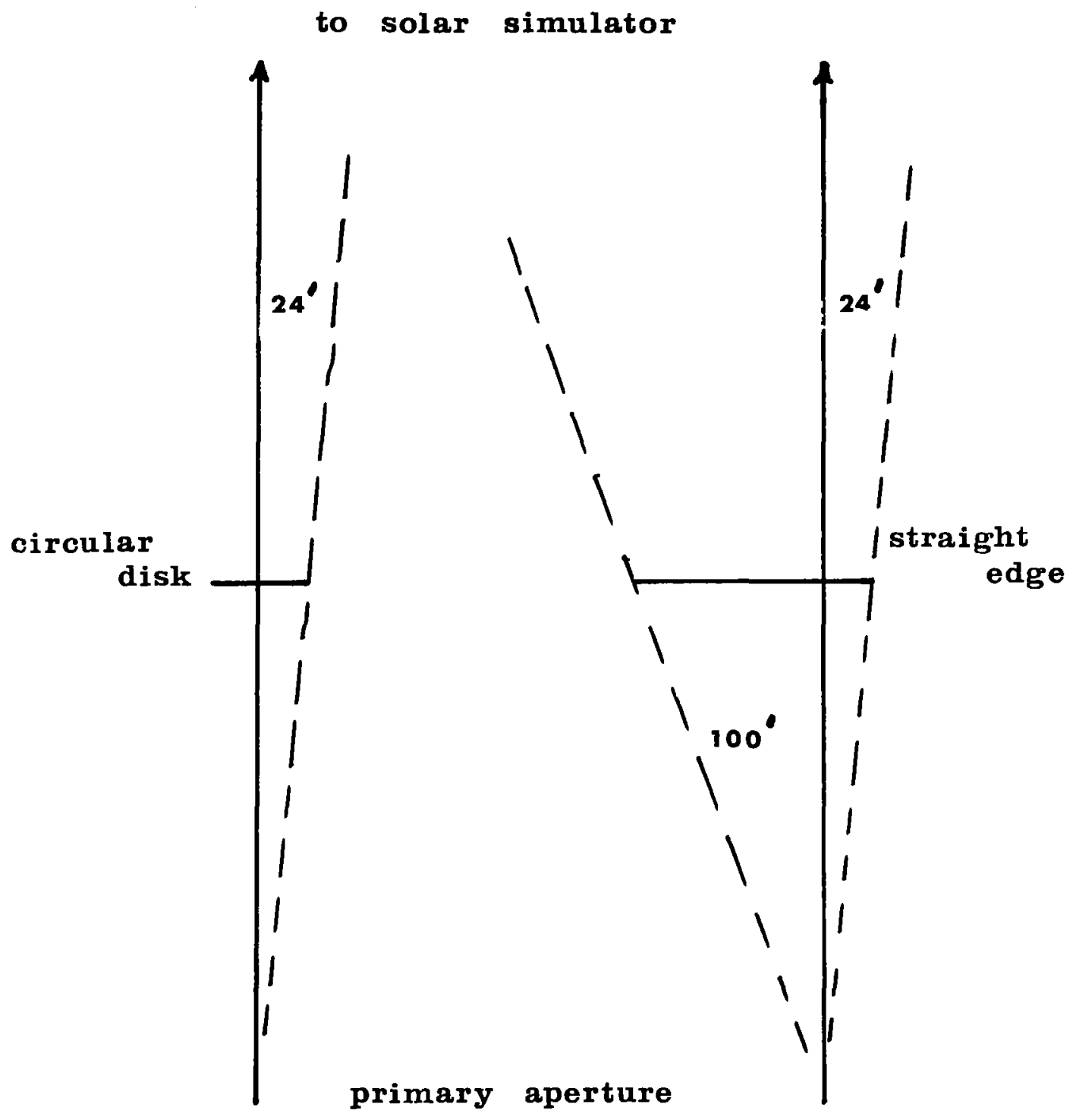

Figure 2.- Geometry of the comparison betweer the diffracted radiance produced by a knife edge and a circular disk. 


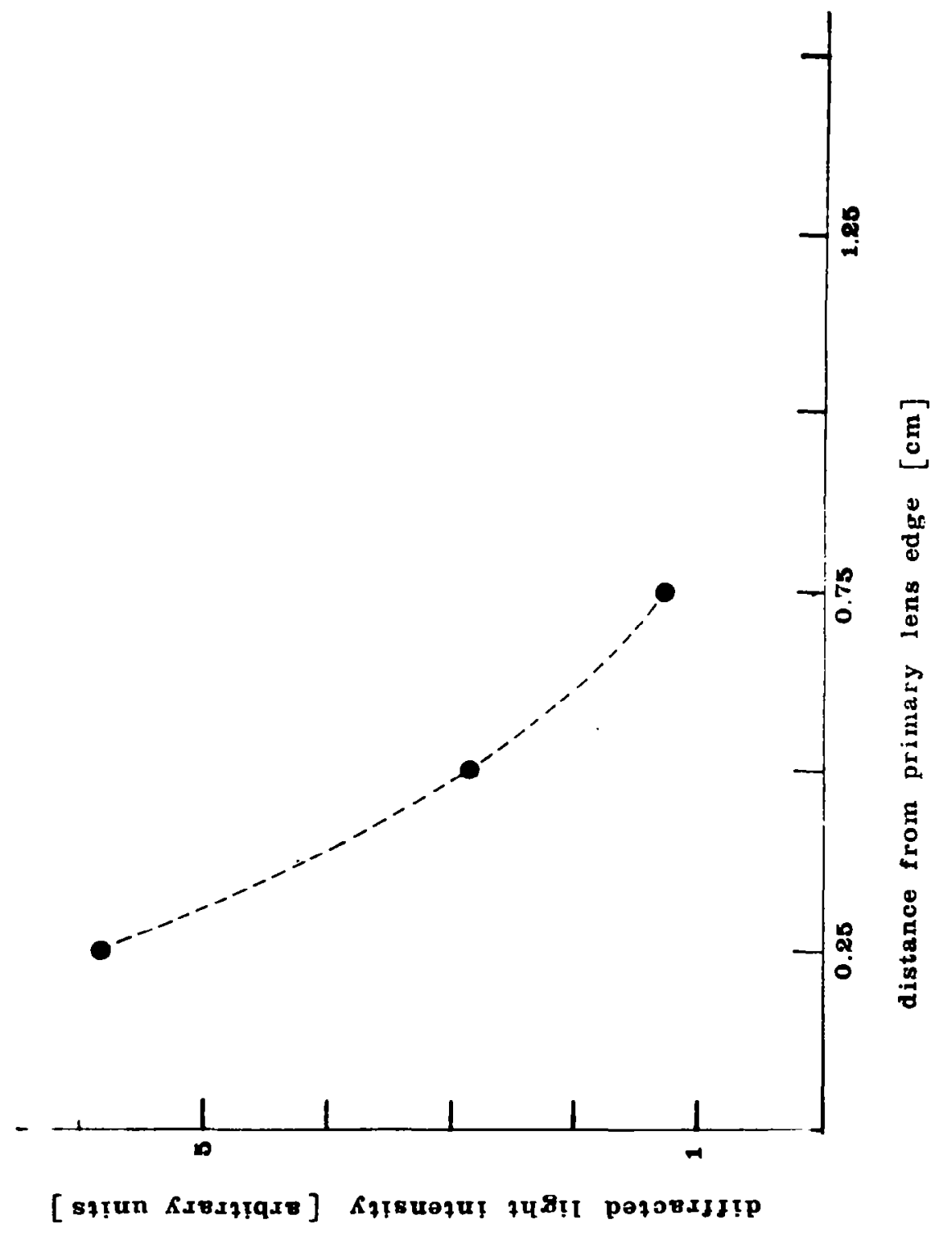

Figure 3.- Observed intensity due to light diffracted from the flight multiple knife edge assembly, across the face of the primary lens aperture. 


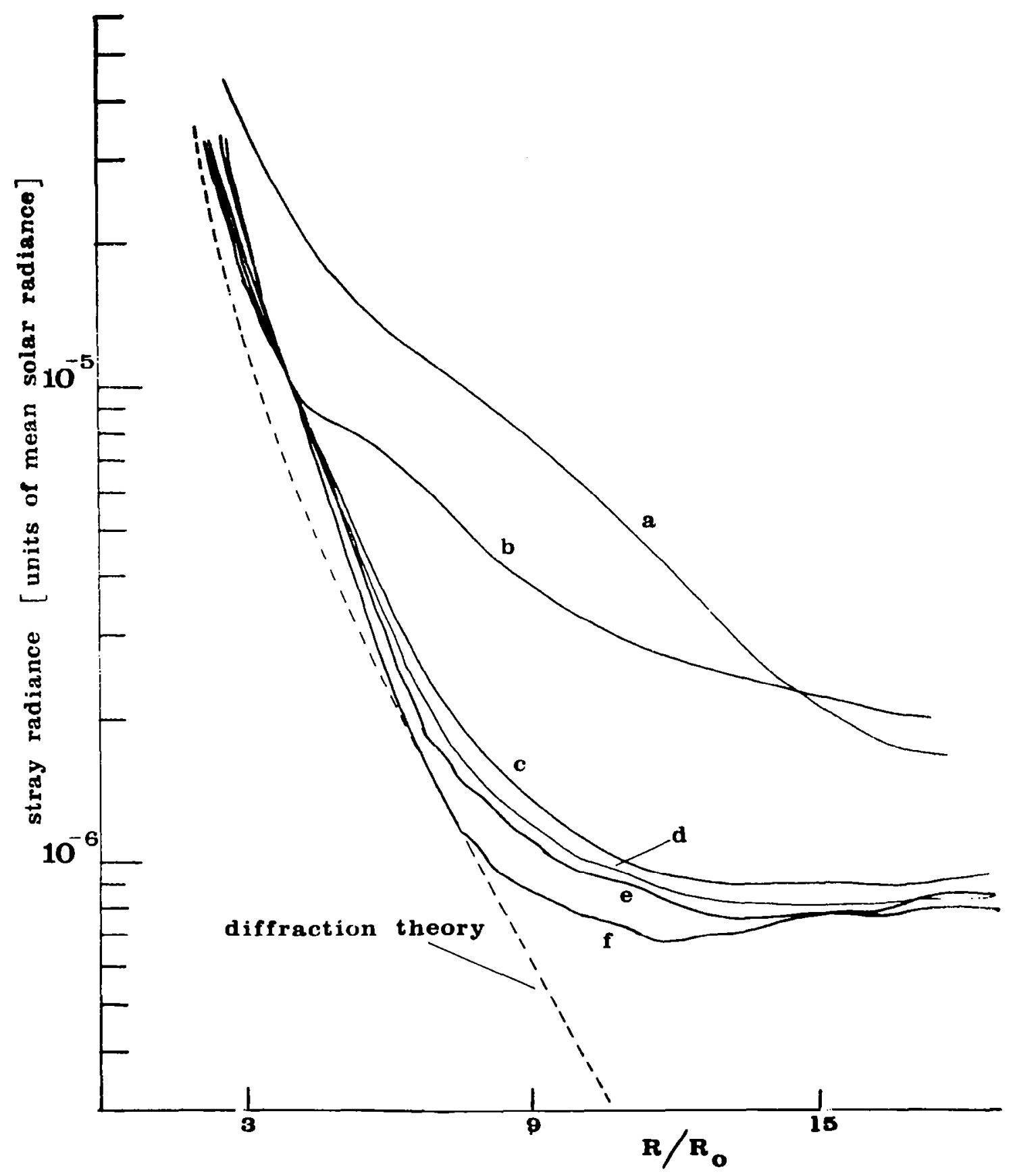

Figure 4.- Stray radiance versus position angle for various types of selected quartz prinary objective lenses. The types are: a-GE Type I5I (sample one); b-Dynasil UV 1000; c-Engelhard Infrasil I; d-GE Type 151 (sample two); e-Fngelhard Homosil; f-Corning Optical. The dashed curve is the calculated diffracted light from a circular aperture, due to a circular source subtending 32 minutes of arc. 


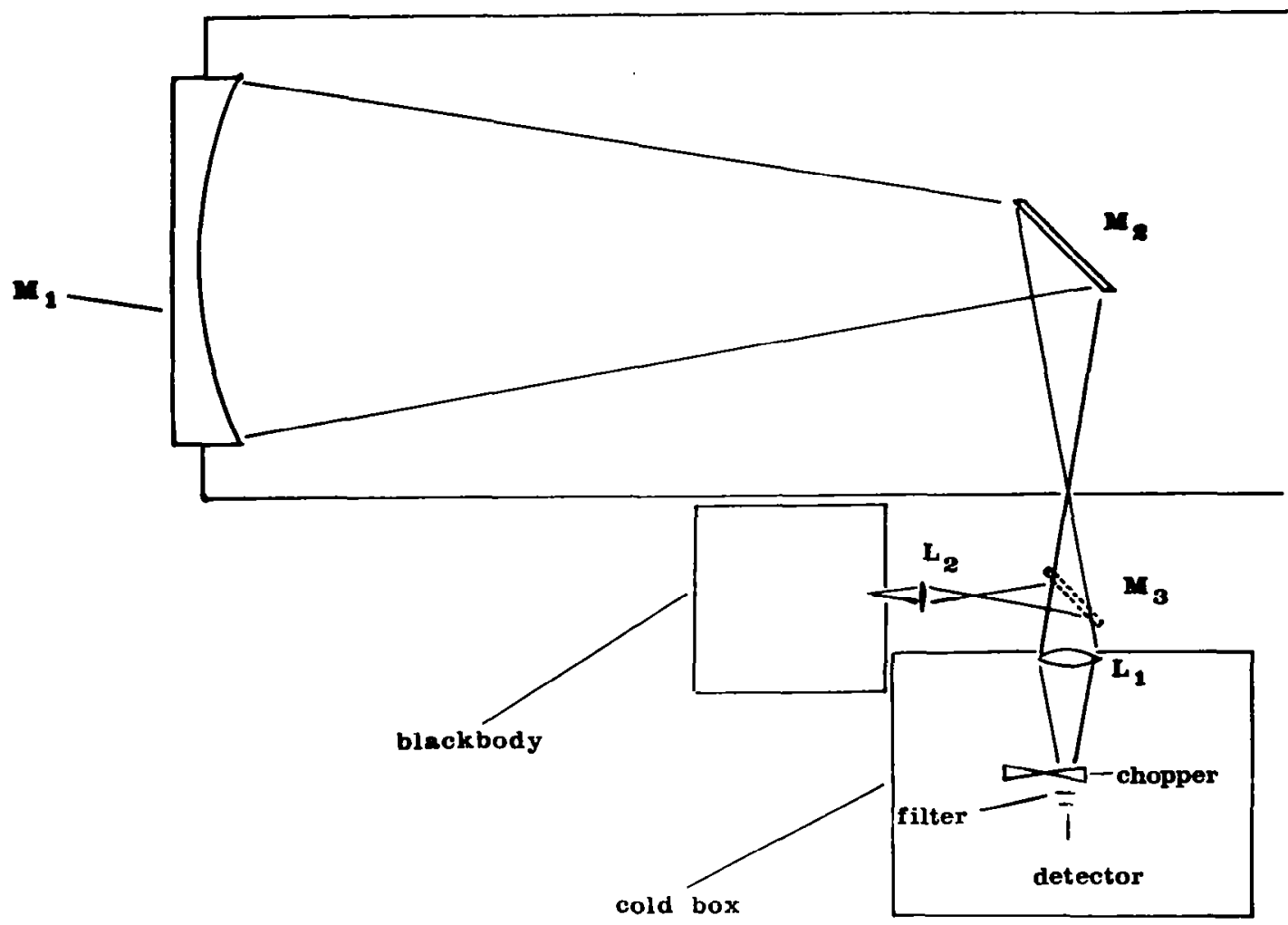

Figure 5.- The infrared eclipse telescope. Mirrur $M_{1}$ is spherical, $29.2 \mathrm{~cm}$ diameter, and $f / 3.1$. Mirror $M_{3}$ may be introduced to direct the radiation from the temperature controlled blackbody source into the detection assembly. Lenses $\mathrm{L}_{1}$ and $\mathrm{I}_{2}$ are made of GE Type 151 quartz. 


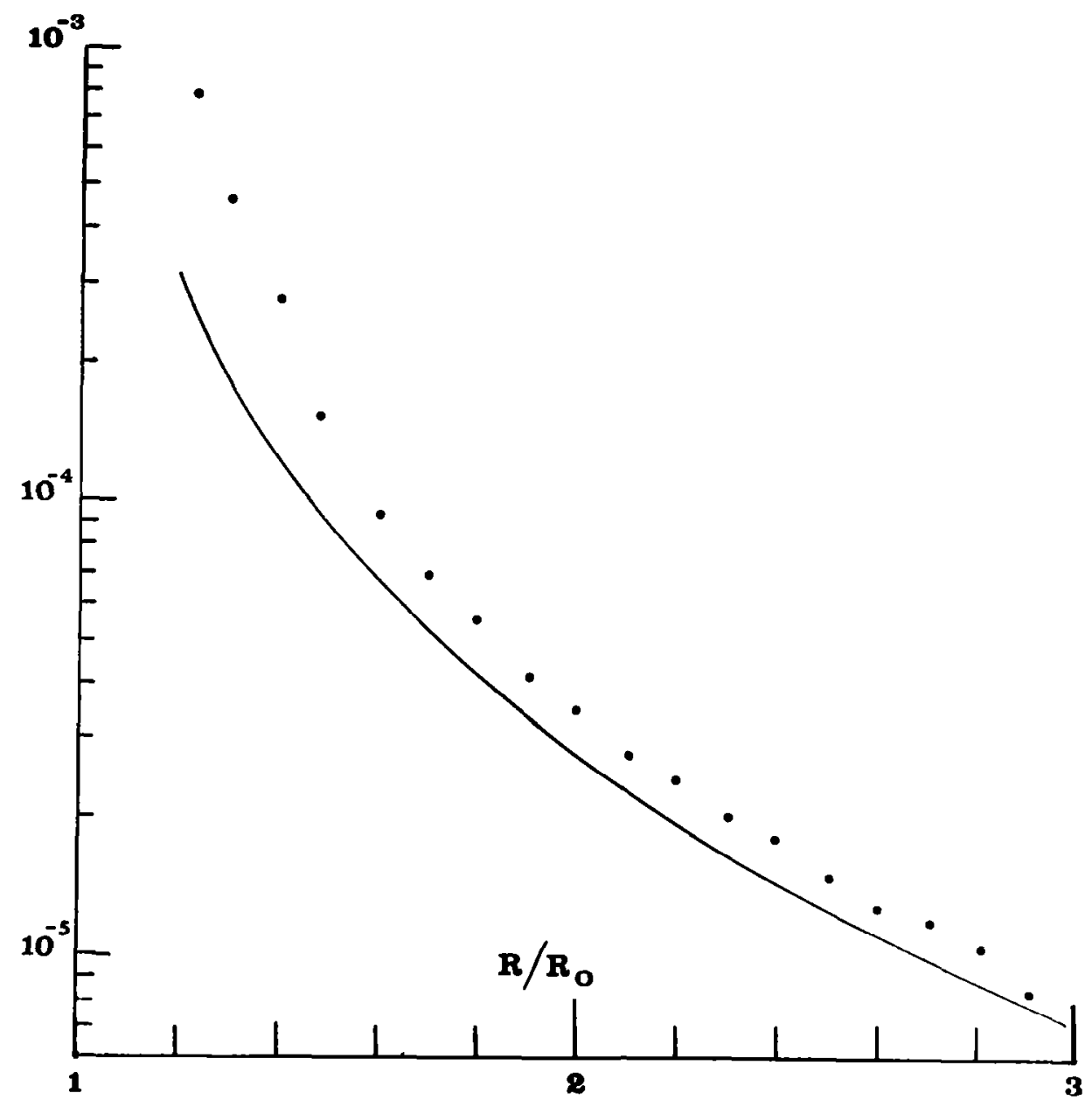

Figure 6.- The measured stray radiance as a function of position angle, for the eclipse telescope. The measured radiance is given in units of that of the simulated solar disk. The solid curve is the calculated diffracted radiance due to a $29.2 \mathrm{~cm}$ diameter circular aperture, for a disk source subtending 32 minutes of arc. 


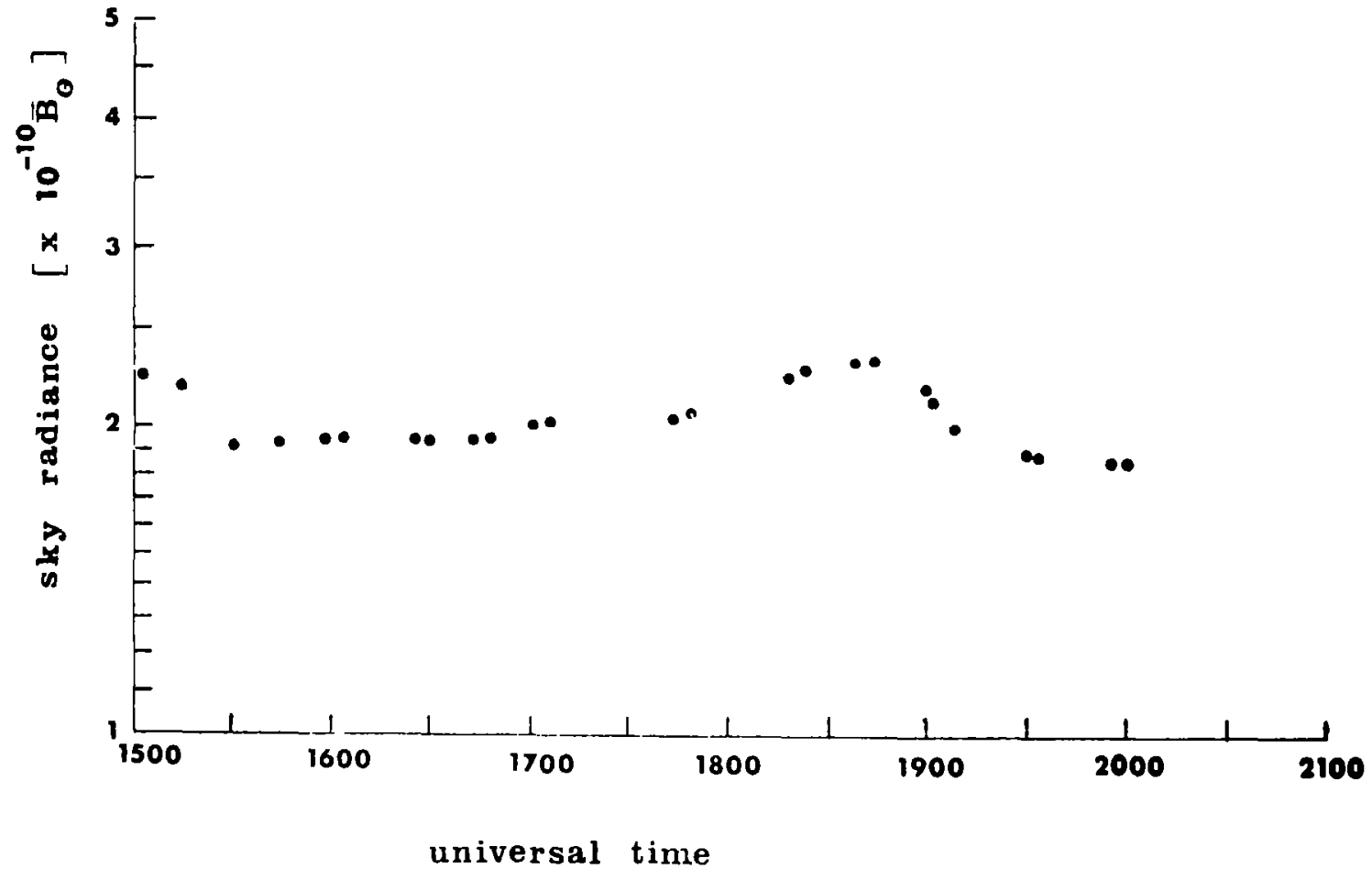

Figure 7.- The $2.2 \mu$ wavelength infrared stratospheric sky radiance, corrected to zenith, observed throughout the duration of the balloon flight of 9 January 1967. The float altitude was $28 \mathrm{~km}$. 


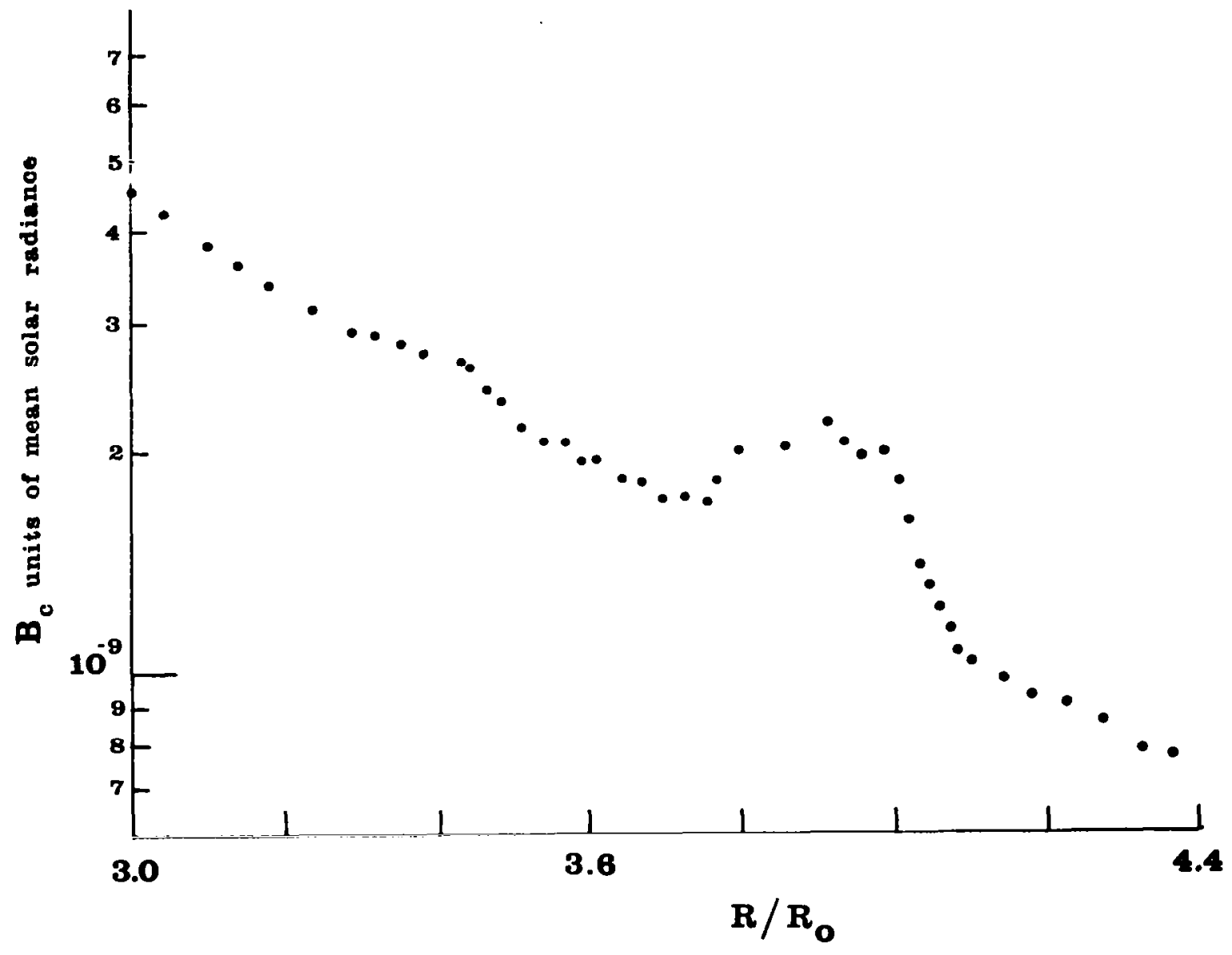

Figure 8.- Thermal emission features observed during the period of totality, 12 November 1966. The plotted points are from two scans, made in the ecliptic plane. 


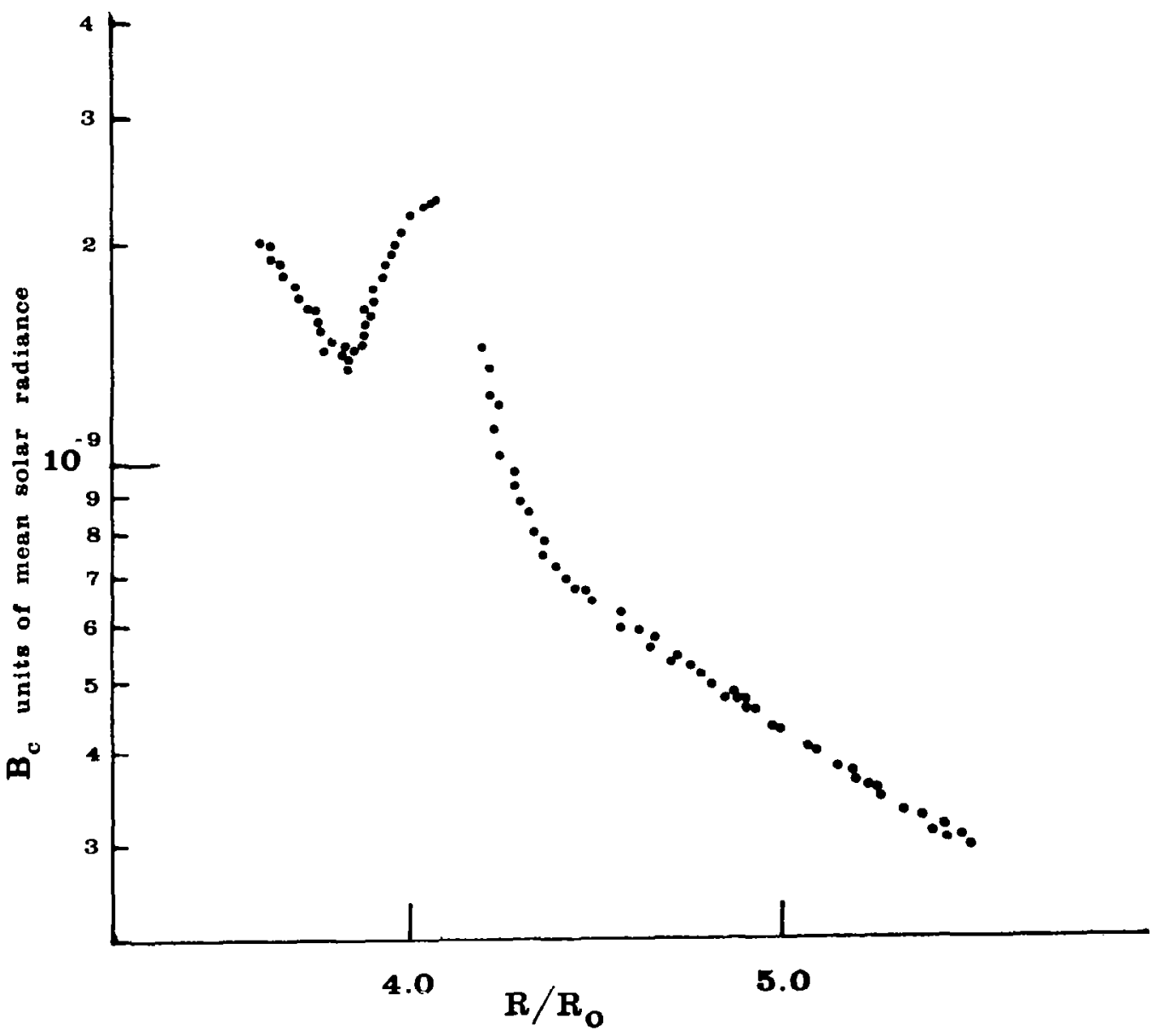

Figure 9.- The thermal emission feature at $4 \mathrm{R}_{0}$, observed during the balloon flight of 9 January 1967. The plotted points are the results of eighteen scans, and are normalized at $4.8 \mathrm{R}_{\mathrm{o}}$. 


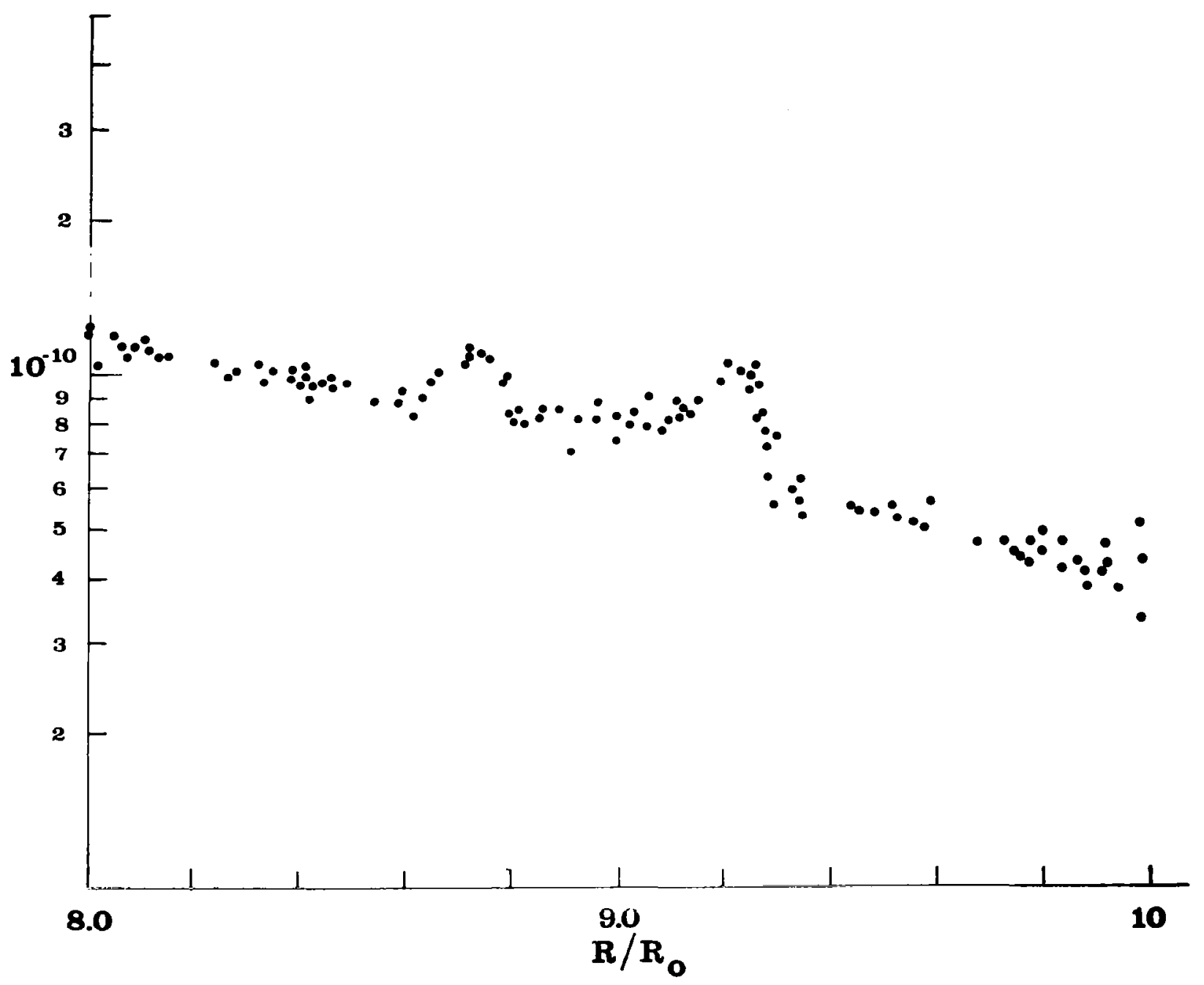

Figure 10.- The thermal emission features at $8.7 \mathrm{R}_{\odot}$ and $9.2 \mathrm{R}_{\odot}$, observed during the balloon flight of 9 January 1967. The plotted points are the results of eighteen scans, and are normalized at $8.0 R_{0}$. The units of the ordinate are those of the mean solar disk radiance, at $2.2 \mu$. 


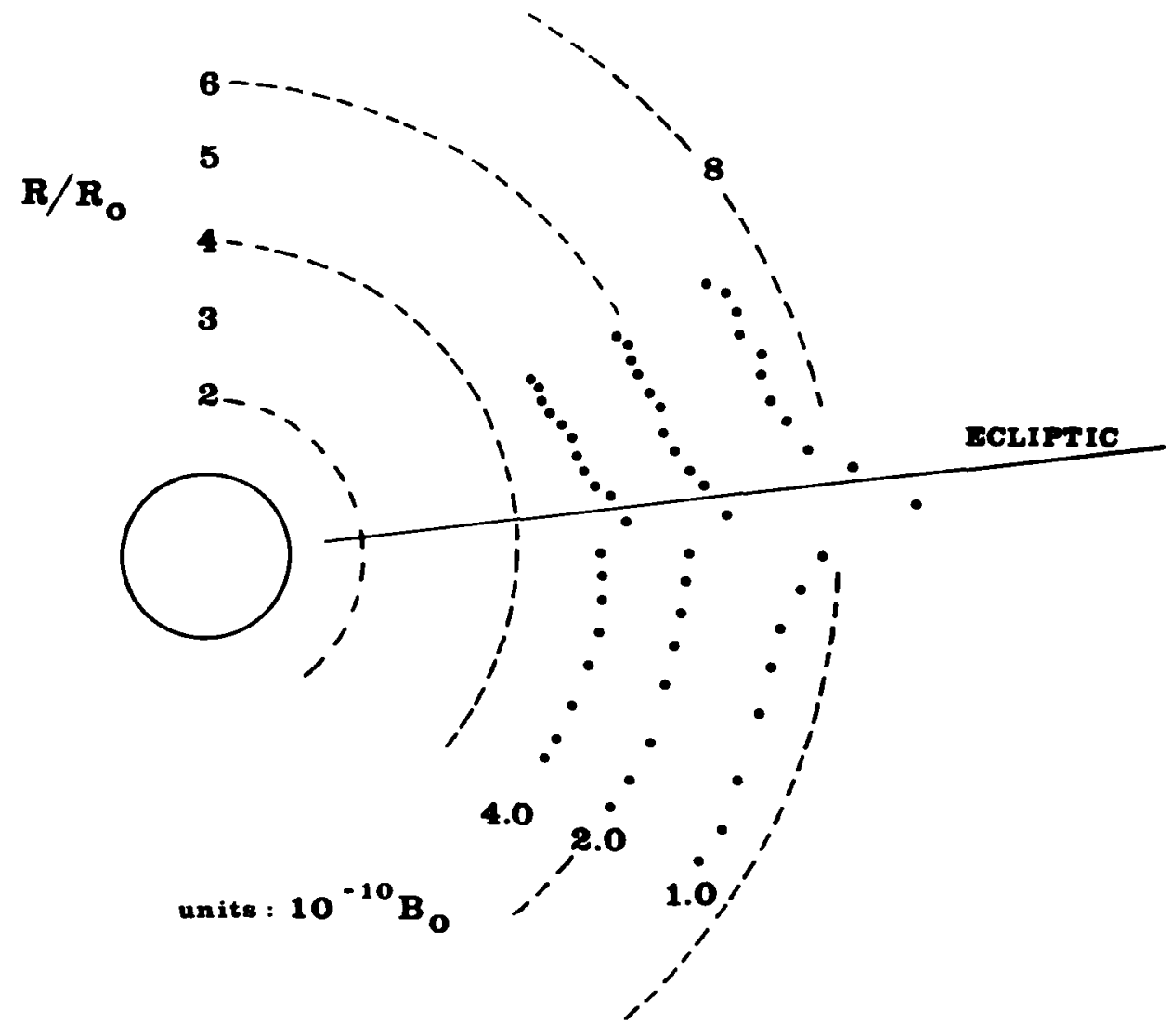

Figure 11.- Infrared coronal isophotes, determined from the balloon flight of 9 January 1967. North is toward the binding, and east to the top of the page. 